\title{
A case report of left ovarian borderline mucinous cystadenomatous cyst containing a neural sarcomatous nodule in an 18 year old female
}

\author{
Nashwa Abulhassan ${ }^{1}$, Tariq Miskry ${ }^{2}$, Mohamed El Mouslemany ${ }^{3}$
}

\author{
${ }^{1}$ Department of Obstetrics \& Gynaecology, Frimley Park Hospital, Surrey, GU16 7UJ, UK \\ ${ }^{2}$ Department of Obstetrics \& Gynaecology, St Mary's Hospital, Paddington, W2 1NY, UK \\ ${ }^{2}$ Department of Obstetrics \& Gynaecology, Ealing Hospital, Southall, UB1 3HW, UK
}

Received: 22 May 2014

Accepted: 10 June 2014

\section{*Correspondence:}

Dr. Nashwa Abulhassan,

E-mail: nashwa_abulhassan@yahoo.com

(C) 2014 Abulhassan $\mathrm{N}$ et al. This is an open-access article distributed under the terms of the Creative Commons Attribution Non-Commercial License, which permits unrestricted non-commercial use, distribution, and reproduction in any medium, provided the original work is properly cited.

\begin{abstract}
This is a unique case of a $13 \mathrm{~cm}$ torted ovarian borderline mucinous cystadenoma with a neural sarcomatous nodule in an 18 year old female. No previous similar cases have been reported in the literature. There are 6 reported cases of pure ovarian osteosarcoma, 5 cases of osteosarcoma arising from an ovarian teratoma, and 2 cases of osteosarcomas of osseous origin (humerus and maxilla) with metastasis to the ovary in the English literature. An 18 year old female presented with a sudden onset of left flank pain radiating to the left loin. She was not examined vaginally as was never sexually active. TA/US showed normal abdomen, a large complex pelvic cyst $107 \mathrm{~mm}$ x $10075 \mathrm{~mm}$ possibly a left dermoid ovarian cyst. CA125, alpha feto- protein and beta HCG were requested and were awaited. Physical examination showed mild tenderness in the left iliac fossa and suprapubically. There was neither guarding nor rigidity. The diagnosis was a torted ovarian cyst and an emergency laparoscopic procedure to investigate the pain was performed. A torted left ovarian cyst occupying the pelvis and upper abdomen $13 \mathrm{~cm} \mathrm{x} 13 \mathrm{~cm}$ in size was found. Left ovary was untorted and an ovarian cystectomy was performed. Intraperitoneal Cystic fluid spillage occurred during retrieval of cyst through the endobag. Peritoneal lavage with $300 \mathrm{ml}$ of fluid was carried out at the end of the procedure. Histology report from the cyst showed a neural nodule which appears to be an osteosarcoma arising in a background of a borderline mucinous ovarian tumour. Following an MDT discussion, she had a laparoscopic left salpingo-oophorectomy with peritoneal washings and omental biopsy. Peritoneal washings did not show malignant cells. Histology report has confirmed absence of residual ovarian tumour and absence of omental deposits. The prognosis of the borderline mucinous tumours is vastly superior to that of a cystadenocarcinoma. Preserving the contralateral uninvolved ovary in a young woman with a borderline mucinous ovarian tumour is the current trend of management; however the prognosis of borderline ovarian mucinous cystadenomas is less certain in the presence of sarcomatous changes. Ovarian osteosarcomas are rare malignancies that commonly occur in old age group. Most patients have advanced stage disease at presentation.
\end{abstract}

Keywords: Ovarian borderline mucinous cystadenoma, Ovarian sarcoma, Ovarian torsion, Rare ovarian malignancies

\section{INTRODUCTION}

This case report of torted ovarian borderline mucinous cystadenoma with a neural sarcomatous nodule in an 18 year old female appears to be a unique with no previous similar cases reported in the literature.
There are 6 reported cases of pure ovarian osteosarcoma, 5 cases of osteosarcoma arising from an ovarian teratoma, and 2 cases of osteosarcomas of osseous origin (humerus and maxilla) with metastasis to the ovary in the 
English literature. The average tumor sizes were 13.46, 13.82 , and $16 \mathrm{~cm}$, respectively.

In 2003, Aygun et al. have reported a case of left ovarian osteosarcoma arising from a mature cystic teratoma in a 14 year old adolescent. ${ }^{2}$ She had a metastatic lesion in the abdomen that did not respond to 2 courses of cisplatin, doxorubicin, ifosfamide, and high-dose methotrexate. Seven months after completion of chemotherapy, there were simultaneous local recurrence and lung metastases.

The category of "ovarian tumors of borderline malignancy" was introduced by the World Health Organization (WHO) in 1971.

The "borderline" terminology was meant to be a provisional one, until detailed studies would reveal definite criteria to classify these tumors as either benign or malignant. ${ }^{3}$ However, no general consensus about the terminology has been achieved, and these tumors continue to cause considerable controversy among the experts. "Borderline" is still an accepted, if not the preferred term by the majority of pathologists and gynaecologic oncologists. ${ }^{4}$

Mucinous cystadenomas make up 15-20\% of all ovarian tumors. They often become very large and can extend up into the abdomen. Pseudomyxoma peritonei can result if the tumour ruptures and spills its contents into the abdomen.

These tumours are usually evaluated using ultrasound, CT scan, or MRI. Findings on imaging studies are nonspecific. These ovarian tumors are usually multiseptated, cystic masses with thin walls. They also contain varying amounts of solid tissue which consists of proliferating stromal tissue, papillae, or malignant tumour cells.

Mucinous cystadenomas are divided into three categories: benign, borderline, and malignant. Survival is largely dependent on the histology of the tumour, with a 10 year survival rate of $100 \%$ for benign tumours, $60 \%$ for borderline tumours, and only $34 \%$ for the malignant subtype.

There is some difference in ages of the peak incidence for the different subtypes with considerable overlap. In general, benign tumours tend to present earlier with a peak age incidence between 30-50 years of age.

Borderline mucinous cystadenomas make up about $10 \%$ of mucinous ovarian neoplasms and are bilateral in $10 \%$ of cases. ${ }^{5}$

\section{CASE REPORT}

An 18 year old female presented to her GP with left flank pain. Pain resolved. USS showed normal abdomen, a large complex pelvic cyst 107 x 100 x $75 \mathrm{~mm}$ possibly a left ovarian dermoid cyst.

Patient was discharged and advised to have an outpatient gynaecology clinic appointment. CA125, alpha fetoprotein and beta HCG were requested by her GP.

4 days later she presented to another Early Pregnancy Unit and was complaining of a sudden onset of left flank pain radiating to the left loin and left iliac fossa. She was not vomiting and had no other symptoms.

She was day 7 of her cycle. Her periods were occurring every 14 days and she bled for 7 days. She gave history of never been sexually active before and pregnancy test was negative.

She was type 1 insulin dependent diabetic. She had a repeat abdominal scan which confirmed the finding of a large complex left ovarian cyst.

On examination her observations were stable. On abdominal examination she had a soft abdomen with mild tenderness in the left iliac fossa and suprapubically. There was neither guarding nor rigidity.

The diagnosis was a torted ovarian cyst and she was admitted, kept nil by mouth and was started on insulin sliding scale. Her blood results were: HB: $13.2 \mathrm{~g} / \mathrm{dl}$, WWC: 7.8, normal U \& E, Normal LFT, amylase: 124, CRP 4.9 Tumour marker results were still being awaited.

She had an emergency laparoscopic procedure to investigate the pain. A torted left ovarian cyst occupying the pelvis and upper abdomen $13 \mathrm{~cm} \mathrm{x} 13 \mathrm{~cm}$ in size was found. Left ovary was untorted and an ovarian cystectomy was carried out. Intraperitoneal cystic fluid spillage occurred during retrieval of cyst through the endobag. Peritoneal lavage was done with $300 \mathrm{ml}$ normal saline.

The sample was sent to histology. She made an eventful recovery and was discharged the next day.

Histology report from the cyst showed: A neural nodule which appears to be an osteosarcoma arising in a background of a borderline mucinous ovarian tumour.

CA125: 37, CEA: 2, CA199: 36

CT pelvis/abdomen/chest and MRI pelvis were organised.

CT thorax/abdomen/pelvis showed a $1.2 \mathrm{~cm}$ nodule in the left axilla. No other abnormality identified in thorax, abdomen and pelvis.

MRI showed Left haematosalpinx. No suspicious ovarian mass. No disease outside the pelvis. The case was discussed at MDT and the decision was to perform 
peritoneal washings, left salpingo-oophorectomy and omental biopsies. She had a laparoscopic left salpingooophorectomy with peritoneal washings and omental biopsy.

Peritoneal washings did not show malignant cells. Histology report has confirmed absence of residual ovarian tumour and absence of omental deposits.

CT PET was organised to clarify the nature of the nodule in left axilla. It did not identify any suspicious site of FDG uptake.

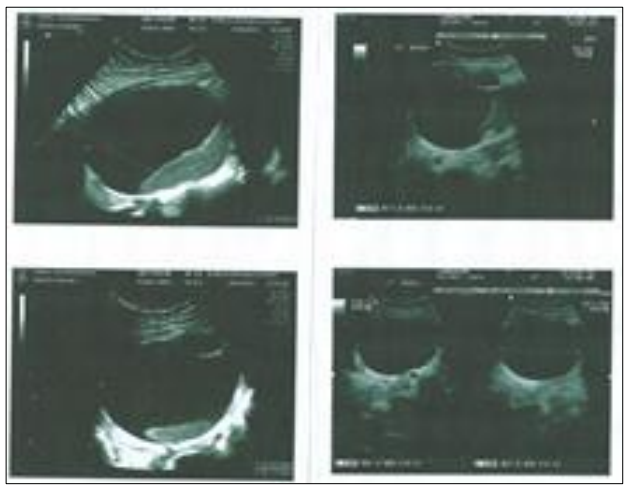

Figure 1: Trans abdominal scan of left ovarian cyst.

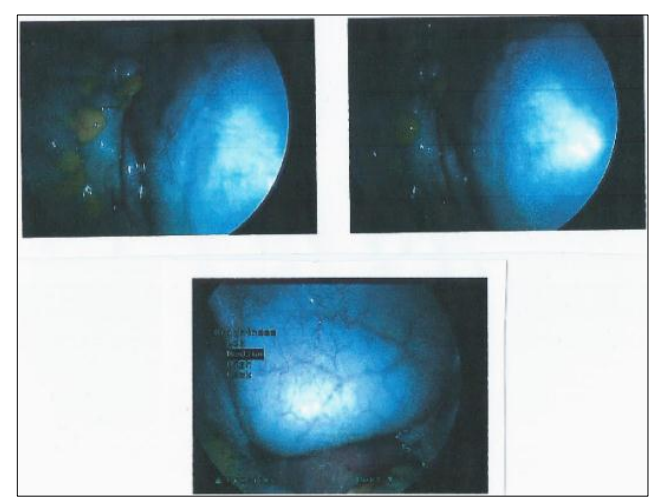

Figure 2: Laparoscopic appearance of large torted left ovarian cyst.

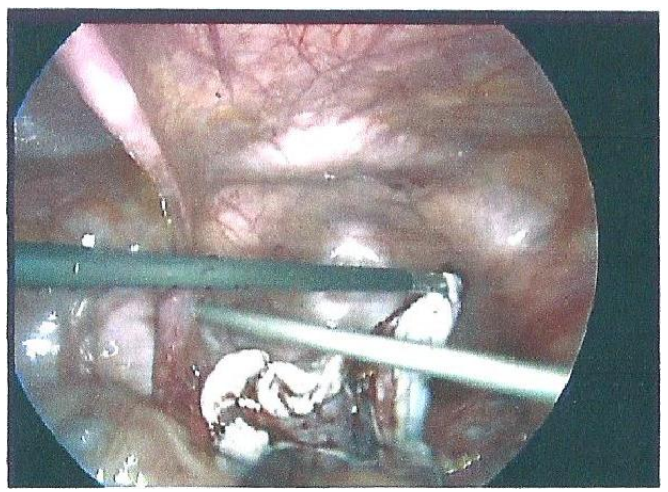

Figure 3: Laparoscopic appearance of left ovary following correction of torsion and cystectomy.

\section{DISCUSSION}

Torsion of ovaries is common in young females aged less than 30 years old. One of the risk factors is presence of an ovarian cyst.

Ovarian tumors, both benign and malignant, are implicated in $50-60 \%$ of cases of torsion. Involved masses are nearly all larger than 4-6 cm, although torsion is still possible with smaller masses.

Dermoid tumors are most common. Malignant tumors are much less likely to result in torsion than benign tumors are. This is because of the presence of cancerous adhesions that fix the ovary to surrounding tissues.

In this case, ovarian torsion occurred in a complex cyst which was thought to be dermoid, however was later histologically proven to be a borderline mucinous cystadenoma with an osteosarcomatous neural nodule.

Intraperitoneal spillage of ovarian cystic contents occurred during retrieval of the cyst. However cytology report from the peritoneal washings taken during the second laparoscopic procedure did not show evidence of malignant cells.

In a review of the literature combining reports of 470 laparoscopic dermoid cystectomies, spillage occurred in 310 cases $(66 \%)$. Major postoperative complications were seen in only 1 case, with chronic granulomatous peritonitis occurring 9 months postoperatively. The conclusion reached was that chemical peritonitis following dermoid spillage during laparoscopic surgery is a rare complication with an incidence of $<1 \%$ if cyst contents are carefully and entirely removed. ${ }^{6}$

Vergote et al. ${ }^{7}$ designed a retrospective study in 2001 including 1545 patients from 6 countries who had epithelial ovarian cancer stage I. They revealed that ruptures before or during surgery were found to be independent and strong prognostic factors.

Controversially, several multivariate analyses did not confirm the risk related to puncture of malignant ovarian tumors. Sevelda et al. ${ }^{8}$ found a 5 -year survival of $76 \%$ in 30 patients with stage I ovarian cancer with capsular rupture and $76 \%$ in 30 similar patients who did not have capsular rupture. The multivariate analysis of 519 patients with stage I epithelial ovarian cancer by Dembo et al. ${ }^{9}$ showed that tumor grade, ascites, and tumor adherence were significant predictors of 5-year diseasefree survival but that tumor rupture was not predictive.

As a result, there is no consensus about the rupture of suspected adnexal masses, but it would be prudent to take every care during surgery to attempt to maintain capsular integrity to minimize any possible risk of tumor dissemination and when deciding whether to puncture, the surgeon has to be alert because there are potential but unknown consequences for the prognosis. 
The prognosis of the borderline mucinous tumours is vastly superior to that of a cystadenocarcinoma. Findings of William et al. ${ }^{10}$ substantiate the rationale for preserving the contralateral uninvolved ovary in a young woman with a borderline mucinous ovarian tumour.

The prognosis is less certain in the presence of sarcomatous changes.

Ovarian osteosarcomas are rare malignancies that commonly occur in old age group with peak incidence between 70-74 years old. Most patients have advanced stage disease at presentation.

Ovarian osteosarcomas have a high $(>60 \%)$ case fatality rate; analysis of the reported cases suggests that the latter is attributable to the advanced stage at which most patients present, as there are no reported cases of patients with stage 1 disease whose diseases have recurred after surgical resection or who have died of their disease.

Funding: No funding sources Conflict of interest: None declared

Ethical approval: Not required

\section{REFERENCES}

1. Fadare $\mathrm{O}$ et al. Primary osteosarcoma of the ovary: a case report and literature review. Int J Gynaecol Pathol. 2007 Jan;26(1):21-5.

2. Aygun et al. Clinical and laboratory observations. J Paediatr Haematol Oncol. 2003 May;25(5):410-3.

3. Fritz MA, Speroff L. Ovarian tumors. In: Fritz MA, Speroff L, eds. Clinical Gynecologic Endocrinology and Infertility. 8th ed. Philadelphia: Lippincott, Williams and Wilkins; 2011: 1221-1248.

4. Schlosshauer. Ovarian tumors of borderline malignancy. In: Liane Deligdisch, Nathan G. Kase, Carmel J. Cohen, eds. Altchek's Diagnosis and Management of Ovarian Disorders. 3rd ed. Cambridge: Cambridge University Press; 2013: 89100.

5. Turkyilmaz E, Korucuoglu U, Kutlusoy F, et al. Recurrent mucinous cystadenoma: a laparoscopic approach. Arch Gynaecol Obstet. 2009 March;279(3):387-9.

6. Nezhat CR, Kalyoncu S, Nezhat CH, Johnson E, Berlanda N, Nezhat F. Laparoscopic management of ovarian dermoid cyst: ten year's experience. J Soc Laparoendosc Surg. 1999;3:179-84.

7. Vergote I, De Brabanter J, Fyles A, et al. Prognostic importance of degree of differentiation and cyst rupture in invasive stage 1 epithelial ovarian carcinoma. Lancet. 2001;357:176-82.

8. Sevelda P, Vavra N, Schemper M, Salzer $H$. Prognostic factors for survival in stage I epithelial ovarian carcinoma. Cancer. 1990;65:2349-52.

9. Dembo AJ, Davy M, Stenwig AE, Berle EJ, Bush RS, Kjorstad K. Prognostic factors in patients with stage I epithelial ovarian cancer. Obstet Gynaecol. 1990;75:263-73.

10. William R. Hart, Henry J. Borderline and malignant mucinous tumors of the ovary. Histologic criteria and clinical behavior. Cancer. 1973 May;31(5):1031-45.

DOI: $10.5455 / 2320-1770$. ijrcog20140904

Cite this article as: Abulhassan N, Miskry T, Mouslemany ME. A case report of left ovarian borderline mucinous cystadenomatous cyst containing a neural sarcomatous nodule in an 18 year old female. Int J Reprod Contracept Obstet Gynecol 2014;3:760-3 